\title{
Shock cardiogénico causado por implantación anómala de arteria coronaria
}

\author{
ALEJANDRO DONOSO F. ${ }^{1}$, PABLO CRUCES R. ${ }^{1}$, \\ FRANCO DÍAZ R. ${ }^{2}$, CRISTIÁN CLAVERÍA C. ${ }^{3}$ \\ 1. Médico. Área de cuidados críticos. Unidad de gestión clínica del niño. Hospital Padre Hurtado. \\ 2. Médico. Programa de medicina intensiva infantil. Universidad del desarrollo Clínica Alemana de Santiago. \\ 3. Cardiólogo. Unidad de gestión clínica del niño. Hospital Padre Hurtado.
}

\begin{abstract}
Cardiogenic shock caused by coronary artery anomalous implantation

Background: Congenital anomalies of coronary implantation are unfrequent, only $1 \%$ of congenital cardiopathies. The anomalous origin of left coronary artery from the pulmonary artery (alcapa) is far more frequent and its manifestations usually arise from myocardial ischemia. Case-report: A male infant 2 month-old that was admitted at our unit with cardiogenic shock and severe metabolic acidosis. The chest X-ray showed cardiomegaly and the echocardiography found diastolic left ventricular dysfunction, with left ventricular shortening fraction of $13 \%$, evidence of dilated cardiomyopathy and anomalous insertion of left coronary artery in the left postero-lateral side of pulmonary artery trunk. Connected to mechanical ventilation, he received crystalloids, hemoderivatives, inotropic hemodynamic support, furosemide and calcium continuous infusion. Moderate hypothermia appeared the second and third day after admission. He developed multiorgan dysfunction with cardiogenic shock and non-oliguric renal failure. At the fifth day, he was stable enough to try surgical repair. Coronary transference from pulmonary artery trunk to aorta was performed without serious adverse events. Finally, a month after his admission, he was discharged home. An ambulatory evaluation 3 months after showed normal cardiac function. Comment: Myocardial ischemia is the main manifestation of alcapa. Its signs and symptoms usually start at the end of the first gestational trimester, according to the lower pulmonary vascular resistance. The clinician must be aware in every patient with cardiogenic shock and dilated cardiomyopathy. Surgical repair must be done, although good outcomes can be achieved only with early and reliable diagnosis.

(Key words: anomalous coronary artery, congenital heart disease, cardiogenic shock).

Rev Chil Pediatr 2008; 79 (5): 509-515
\end{abstract}

\section{RESUMEN}

Introducción: Las anomalías de implantación de las arterias coronarias constituyen menos del 1\% de las cardiopatías congénitas. La implantación anómala de la arteria coronaria izquierda (IAACI) es la más frecuente y se presenta habitualmente con manifestaciones de isquemia miocárdica. Caso clínico: Comunicamos un lactante de 2 meses de edad, sexo masculino, quién ingresó con shock cardiogénico y acidosis metabólica grave. Radiografía de tórax con cardiomegalia marcada, ecocardiograma reveló disfunción sistólica ventricular izquierda grave con FAVI 13\%, evidencias de miocardiopatía dilatada y sospecha

Trabajo recibido el 10 de marzo de 2008, devuelto para corregir el 12 de mayo de 2008, segunda versión el 16 de mayo de 2008, aceptado para publicación el 04 de junio de 2008.

Correspondencia a:

Dr. Alejandro Donoso F.

E-mail: adonoso@hurtadohosp.cl 
IAACI (cara posterolateral izquierda tronco arteria pulmonar). Recibió ventilación mecánica, expansores de volumen, transfusión de hemoderivados, drogas inotrópicas, infusión furosemida, hipotermia moderada e infusión de calcio continua. Evolucionó con disfunción orgánica con: shock cardiogénico y falla renal no oligúrica. Al quinto día se logra estabilización y se somete a resolución quirúrgica, efectuándose transferencia coronaria desde tronco de arteria pulmonar a aorta. Fue dado de alta a su domicilio al mes de ingresado. Control ambulatorio a los tres meses con función cardíaca normal. Comentario: IAAC se manifiesta por isquemia miocárdica y los síntomas aparecen habitualmente al ocurrir el descenso de la resistencia vascular pulmonar. El resultado a largo plazo es bueno, si se realiza la revascularización en forma temprana. Su sospecha y búsqueda debe de ser acuciosa, contando con el valioso rol de la evaluación ecocardiográfica, en todo paciente que curse con shock cardiogénico y miocardiopatía dilatada. (Palabras clave: Arteria coronaria anómala, cardiopatía congénita, shock cardiogénico).

Rev Chil Pediatr 2008; 79 (5): 509-515

\section{Introducción}

Las anomalías congénitas de las arterias coronarias representan el $2 \%$ de las cardiopatías congénitas, siendo la implantación anómala de la arteria coronaria izquierda (IAACI) la más frecuente, estimándose su incidencia en 1 en 300000 nacidos vivos ${ }^{1-3}$. Hace algunas décadas el diagnóstico de IAACI, también conocido como síndrome de Bland-White-Garland, era casi exclusivo post-mortem, pero con la llegada de técnicas no invasivas es posible un diagnóstico precoz y tan certero que en ocasiones no es necesario utilizar el gold standard, la angiografía ${ }^{4}$. Las manifestaciones clínicas de esta patología se derivan de la isquemia miocárdica, habitualmente manifestada al fin del primer trimestre de vida por los cambios fisiológicos propios en la hemodinamia del lactante menor ${ }^{5}$. Se estima que el $85 \%$ de los pacientes inicia sus síntomas antes del año de vida, pero éstos son inespecíficos constituyendo un franco dilema diagnóstico ${ }^{3,6}$. Tal como lo describiera Bland y cols en su reporte original en 1933, se caracteriza por llanto episódico, rechazo alimentación y disnea $^{2,7}$. Dependiendo del grado de isquemia, extensión y tiempo de evolución se desarrolla insuficiencia cardíaca congestiva, insuficiencia mitral, infarto agudo del miocardio o arritmias de curso fatal ${ }^{2,6,8,9}$. Un pequeño porcentaje, no mayor al 10\% de los casos, puede ser oligosintomático y superar los síntomas del período de lactante gracias al desarrollo de arterias colaterales, y llegar a la adultez ${ }^{10,11}$. En este grupo etáreo se diagnostican como hallazgos en pacientes con síndrome anginoso asociado a comorbilidades menores o ausentes, lo que tradu- ce una reserva coronaria disminuida ${ }^{6}$. En niños mayores y adolescentes recientemente se ha asociado a muerte súbita ${ }^{2,5}$.

El objetivo de la presente revisión es reportar un caso clínico de IAACI que se presentó como un shock cardiogénico y describir la fisiopatología, las formas de presentación, diagnóstico y tratamiento de esta enfermedad.

\section{Caso clínico}

Paciente sexo masculino de 2 meses de edad. Sin antecedentes perinatológicos significativos. Historia de un mes de dificultad respiratoria catalogada como bronconeumonía y "Síndrome Apneico", las cuales motivaron hospitalización, sin llegar a un diagnóstico definitivo. En aquella oportunidad dentro del estudio se efectuó ecocardiograma el cual reveló insuficiencia mitral leve, no efectuándose mayor estudio.

El día previo a su ingreso presentó episodios de irritabilidad, que motivó una consulta en su hospital de origen. Con evidencias francas de hipoperfusión periférica y dificultad respiratoria en el servicio de urgencia, presentó paro cardiorrespiratorio, reanimado en forma exitosa. Sus signos vitales al momento de ingresar al área de cuidados críticos de nuestro hospital eran: frecuencia cardíaca: 175/min, Presión Arterial Media: $40 \mathrm{mmHg}$, índice de shock (frecuencia cardíaca/presión arterial sistólica) de 2.9. Al examen físico destacó la presencia de un soplo holosistólico II/VI y hepatomegalia 5 cm BRC. Radiografía de tórax con cardiomegalia marcada (figura 1), ECG con alteración en la repolarización, ausencia de onda q (-) en 
AVL. Ecocardiograma reveló disfunción sistólica ventricular izquierda grave (FAVI 13\%), evidencias de miocardiopatía dilatada y sospecha IAACI (cara posterolateral izquierda tronco AP).

En sus exámenes de laboratorio destacaban: gasometría con $\mathrm{pH}$ en 6,9 y Exceso de Base (BE) -25, saturación venosa central de $\mathrm{O}_{2}$ $\left(\mathrm{SvO}_{2}\right) 42 \%$, lactato en $4,1 \mathrm{mmol} / \mathrm{l}(\mathrm{VN}:<1,7$ $\mathrm{mmol} / 1$ ), hematocrito $22 \%$. Creatinkinasa: 558 , Troponina T: 0,375 ng/ml (VN: <0,05 ng/ml). Conectado a ventilación mecánica convencional, recibió expansores de volumen $75 \mathrm{ml} / \mathrm{kg}$ en

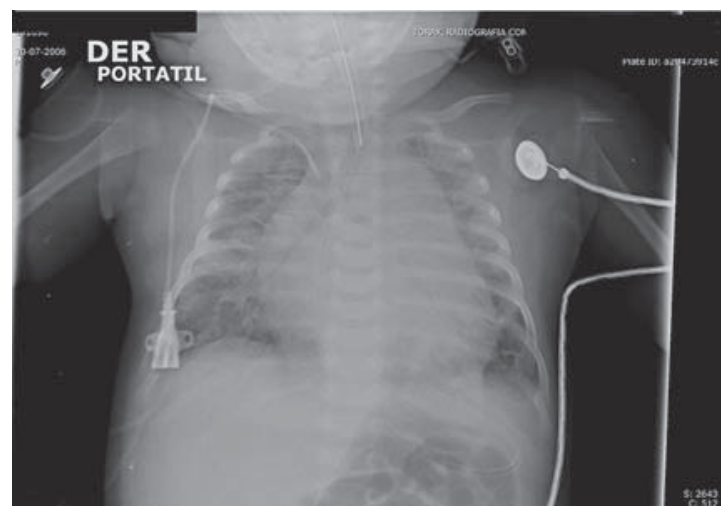

Figura 1. Radiografía de tórax anteroposterior de paciente al ingreso a la Unidad de Cuidados Intensivos. Se observa cardiomegalia significativa para la edad (índice cardiotorácico de $68 \%$ ) e imágenes de relleno alveolar, compatible edema pulmonar agudo. Tubo endotraqueal in situ y catéter venoso central subclavio derecho.

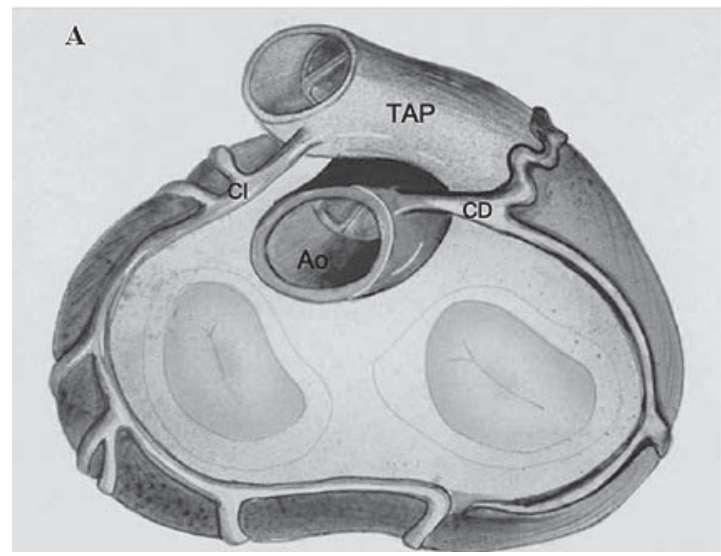

24 horas, transfusión de hemoderivados, drogas inotrópicas (epinefrina $0,3 \mathrm{mcg} / \mathrm{kg} / \mathrm{min}$-dopamina $12 \mathrm{mcg} / \mathrm{kg} / \mathrm{min}$-milrinona $0,5 \mathrm{mcg} / \mathrm{kg} / \mathrm{min}$ ) para estabilizar macrohemodinamia. Cursó con oliguria marcada en las primeras 6 horas que requirió infusión furosemida para débito urinario $2 \mathrm{ml} / \mathrm{kg} / \mathrm{h}$. Se inició hipotermia terapéutica moderada $\left(33-35^{\circ} \mathrm{C}\right)$ e infusión de calcio continua para lograr metas de reanimación metabólicas. En las 24 horas siguientes se logró ascenso de $\mathrm{SvO}_{2}>70 \%$ y corrección progresiva de acidosis metabólica. Ante sospecha inicial de miocarditis viral, recibió infusión de gammaglobulina $2 \mathrm{~g} / \mathrm{kg}$, a las $48 \mathrm{~h}$ se obtuvo PCR para enterovirus negativa.

Evolucionó con disfunción orgánica: shock cardiogénico y falla renal no oligúrica. Al tercer día de ingresado, continuó con falla de bomba (FAVI 17\%), y flujo diastólico reverso en ecocardiografía. La hipotermia fue suspendida en forma gradual a las 72 horas de ingresado y se mantuvo el apoyo con inótropos. Al quinto día se logró estabilización y se sometió a resolución quirúrgica, efectuándose transferencia coronaria desde tronco de arteria pulmonar a aorta (clamp aórtico de 66 minutos, circulación extracorpórea de 170 minutos). La figura 2 muestra una representación esquemática del defecto y su reparación. Al decimosegundo día post operatorio se cerró de esternotomía. Se extubó a los 17 días post operado. Fue dado de alta al mes de ingre-

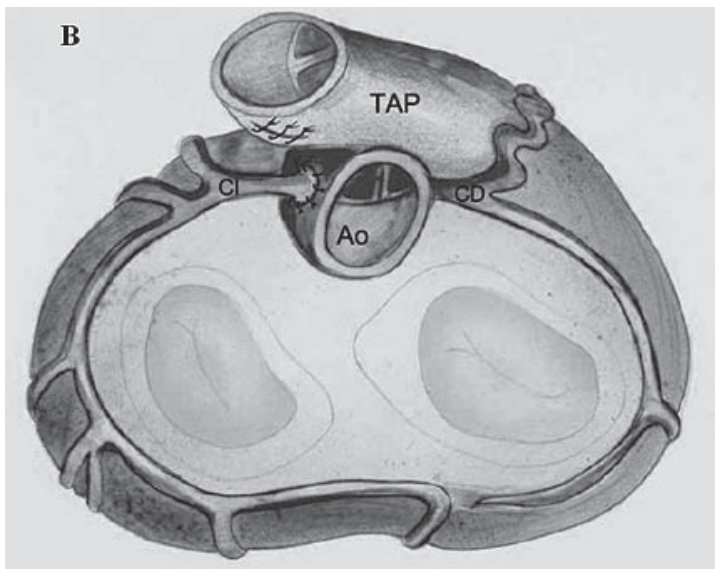

Figura 2. Dibujo esquemático, transección a nivel Auricular visto desde la base del corazón. Ao: Aorta, TAP: Tronco Arteria Pulmonar; CI: Coronaria izquierda; CD: Coronaria Derecha. A) Inserción Anómala de Arteria CI desde TAP. B) Reimplantación de Arteria CI desde TAP a Ao. 
sado, en tratamiento con digital, captoprilo y furosemida. Control de ecocardiograma con ACI desde seno coronario, FAVI 34\% y FE 70\% a los 3 meses. Al año de seguimiento con desarrollo psicomotor y pondo estatural normales.

\section{Discusión}

Las anomalías del árbol coronario pueden ser únicas (primarias) o asociadas a una anatomía anormal de las cavidades cardíacas y vasos sanguíneos mayores (secundarias) ${ }^{3,12}$. Se han descrito diversas malformaciones congénitas de las arterias coronarias en cuanto a origen, número y trayecto, pudiendo ser agrupadas en menores, si el origen de las arterias coronarias es aórtico y mayores cuando hay una mezcla de sangre oxigenada/no oxigenada al existir conexiones anormales (seno coronario, vena cava superior, cámaras cardíacas), así como el origen desde la arteria pulmonar ${ }^{2,3}$. Desde el punto de vista fisiopatológico, es mejor clasificarlas en si presentan o no una alteración hemodinámica significativa ${ }^{3,6}$.

La malformación más frecuente es la IAACI, conocido también como ALCAPA por su acrónimo en inglés (anomalous left coronary artery from the pulmonary artery), representa el $0,5 \%$ de todas las cardiopatías congéni$\operatorname{tas}^{2,3}$.

El primer reporte fue hecho en 1933, por Bland, White y Garland, de ahí su epónimo, en la necropsia de un lactante de 3 meses y medio que presentaba crisis de palidez, disnea y sudoración ${ }^{7}$.

\section{Fisiopatología}

En la vida embrionaria, las arterias coronarias se desarrollan durante la séptima semana de gestación como dos botones endoteliales a ambos lados del tronco arterial común, antes de su división por un septo espiroideo en aorta y arteria pulmonar ${ }^{1,13}$. La IAACI se puede explicar por varias teorías, como la separación anómala del tronco arterial incluyendo el crecimiento de la arteria coronaria izquierda en lo que será la arteria pulmonar, la ubicación incorrecta del primordio que origina la ACI en el territorio de la arteria pulmonar y la posibilidad de que aorta y arteria pulmonar puedan generar yemas de las que se forman las arterias coronarias $^{8}$.

En el momento del nacimiento, la resistencia y presión de la arteria pulmonar es elevada ocasionando una adecuada irrigación del territorio coronario. Mecanismos compensatorios locales para la oxigenación tisular, a pesar de ser sangre con bajo contenido de oxígeno (desaturada) en ACI, logran mantener la vitalidad del tejido. Sin embargo, en la etapa post natal, debido a la caída de la presión de arteria pulmonar, la irrigación del territorio de la ACI se hace insuficiente, limitando la presión de perfusión miocárdica ${ }^{12}$.

\section{Clínica}

Los síntomas de IAACI son derivados de la isquemia de éste territorio arterial. Dependiendo de la edad del paciente es posible agruparla en:

1. Forma infantil o del lactante. Constituye el $85 \%$ de los casos, con síntomas recurrentes y progresivos luego de la etapa neonatal, paralelo a la caída de la resistencia del territorio vascular pulmonar. No hay un adecuado desarrollo de colaterales por lo que hay una escasa reserva coronaria ${ }^{1,9,10}$. Por lo tanto, ante esfuerzos habituales como alimentación o llanto se desarrolla isquemia. En este grupo etáreo los equivalentes anginosos o episodios recurrentes de dolor torácico son de muy difícil diagnóstico, ya que se manifiestan como palidez, sudoración e irritabilidad, muchas veces ausentes al momento de evaluación médica ambulatoria ${ }^{9}$. Todo esto lleva a una insuficiencia cardíaca asociada o no a miocardiopatía dilatada. Su pronóstico es malo y con elevada mortalidad en el primer año de vida, hasta un $65 \%$ a $90 \%$ de no recibir tratamiento oportuno, principalmente por un diagnóstico tardío y de las complicaciones derivadas de la cirugía como arritmias secundarias a la isquemia miocárdica preoperatoria, sindrome de hipodébito, eventual cirugía valvular mitral a futuro o de un inadecuado crecimiento de la anastomosis coronaria (poco probable). Al examen físico una taquicardia persistente asociado a polipnea debido a congestión pulmonar, y en etapas más avanzadas los signos clásicos de insuficiencia cardíaca como hepatomegalia. Es importante recordar que uno de los hallazgos al examen físico es la aparición de un soplo 
diastólico producto de insuficiencia mitral secundaria a isquemia de los músculos papilares, dependientes de la $\mathrm{ACI}^{5,12,13}$.

2. Forma de niños mayores y del adulto. El desarrollo de colaterales y anastomosis intercoronarias permite mantener la función miocárdica y sobrevivir la etapa de la infancia. Habitualmente asintomático, se puede pesquisar por cardiomegalia en la radiografía de tórax o la presencia de soplo cardíaco ${ }^{10}$. Sin embargo, en caso de estar presentes, las manifestaciones clínicas son catastróficas: infarto del miocardio o muerte súbita durante el ejercicio (arritmias ventriculares). En población adulta hay cada vez más reportes de pacientes jóvenes con síndrome coronario agudo sin factores de riesgo importante. Es así como en una reciente publicación basso y cols, señalan que entre un 5 a $35 \%$ de las muertes súbitas en pacientes menores de 35 años son ocasionadas por malformaciones congénitas de las arterias coronarias $^{2,6,10}$.

\section{Diagnóstico}

La ecocardiografía Doppler es una adecuada opción no invasiva para el diagnóstico de IAACI asociada o no a miocardiopatía dilatada. El diagnóstico es definitivo si en el modo 2D se puede observar el lugar de inserción de la ACI en el TAP, lo que habitualmente no es frecuente de observar ${ }^{4,14}$. Así, usualmente otros signos indirectos, aunque no concluyentes, deben ser evaluados, como la dilatación compensatoria de la arteria coronaria derecha (ACD), la relación ACD: anillo aórtico (la cual está aumentada), mayor ecogenicidad de los músculos papilares, la presencia de colaterales y el flujo turbulento en el sitio de conexión en el TAP en el Doppler color ${ }^{4,14}$. Es posible encontrar la presencia de flujo diastólico reverso en ACI, debido a la diferencia de presiones entre la ACD dependiente de Ao y ACI dependiente de TAP, conectados en serie a través de colaterales. Si bien estos hallazgos son poco específicos, su presencia debe hacer sospechar IAACI, más aún en ausencia de visualización óptima del sitio de inserción coronaria. Signos de insuficiencia ventricular como hipokinesia global o segmentaria, fracción de acortamiento ventrí- culo izquierdo (FAVI) disminuida y volúmenes telediastólicos elevados pueden ser observados evidenciando el grado de disfunción cardíaca. A pesar que las alteraciones de la válvula mitral no siempre están presentes, la presencia de hiperecogenicidad debido a cambios fibróticos en los músculos papilares y la cordae tendinea deben hacer sospechar isquemia, especialmente si están asociados a prolapso valvular mitral o insuficiencia valvular ${ }^{4,8,14}$.

El cateterismo puede demostrar la existencia de un cortocircuito izquierda derecha y la angiografía corrobora el diagnóstico anatómico. En la aortografía supravalvular se observa una ACD dilatada, en ausencia del ostium de $\mathrm{ACI}^{3,6}$. En secuencias tardías se puede observar el contraste de la ADA y la circunfleja en forma retrogada por colaterales, en ocasiones llegando hasta el TAP si el flujo es suficientemente grande ${ }^{3,6,15}$. En la actualidad la indicación de angiografía esta indicada sólo en casos de diagnóstico no concluyente ecocardiografíco, anatomía muy alterada y ante la sospecha de otras patologías asociadas.

En los últimos 10 años con el perfeccionamiento de nuevas tecnologías, se han descrito métodos diagnósticos no invasivos prometedores como angioTAC multicorte ${ }^{16} \mathrm{o}$ la angioresonancia coronaria ${ }^{17}$, pero aún limitados a adultos. Los estudios topográficos de viabilidad miocardica en niños, como ecocardiografía de stress, angiotac coronario y estudios de medicina nuclear, no son recomendados ya que no cambian la conducta terapéutica ${ }^{18}$.

El electrocardiograma puede evidenciar signos de isquemia aguda o bien infarto antiguo. Ondas Q patológicas asociadas a T invertidas en las derivaciones I y aVL deben ser buscadas en forma dirigida ${ }^{4,19}$. Otros signos pueden ser visualizados como desviación del eje a izquierda, hipertrofia VI y falta de progresión de onda $\mathrm{r}$ en derivaciones precordiales imitando un IAM lateral ${ }^{4,6}$.

\section{Tratamiento}

El tratamiento es quirúrgico, siendo el objetivo de la terapia médica sólo soporte y estabilización previa a la corrección definitiva del defecto. En nuestro caso el paciente debutó con 
un shock cardiogénico grave. No obstante al igual que otros escenarios de shock, la reanimación inicial con volumen y drogas vasoactivas es vital en un inicio. Sin embargo, el reconocimiento precoz de la falla de bomba es fundamental, ya que a diferencia de lo que ocurre en el shock hipovolémico, los mecanismos compensatorios neurohumorales en este escenario son deletéreos, comprometiendo progresivamente la función ventricular izquierda, aumentando así el trabajo y consumo de oxígeno. Entonces la estrategia terapéutica debe estar enfocada a maximizar la entrega tisular de oxígeno, disminuir la demanda tisular de oxígeno, pero concomitantemente lograr minimizar la demanda miocárdica de oxígeno. La optimización de la precarga debe ser cautelosa, pero no debe demorar bajo ningún supuesto la reanimación inicial. Inicialmente la optimización de la función sistólica debe ser con inótropos como dopamina y/o epinefrina, a los que luego se pueden asociar drogas con acción inodilatadora como la milrinona (siempre con una precarga adecuada) y finalmente en algunos casos puede ser útil el empleo vasodilatadores específicos. El papel que juega la hemoglobina es fundamental en la entrega de oxígeno, por lo tanto la corrección de la anemia, como las eventuales alteraciones electrolíticas es fundamental (calcemia, kalemia). La normalización progresiva de la macrohemodinamia, evaluada por la perfusión clínica, diuresis horaria, depuración de lactato y el estado acido-base señalarán la compensación de la función cardíaca. Idealmente estas deben de presentar un correlato con la metas de reanimación metabólica como son la diferencia arteriovenosa de $\mathrm{Co}_{2}$ y el nivel de saturación venosa central $\left(\mathrm{SvO}_{2}\right)$. En nuestro paciente a pesar de las medidas tomadas, la hipotensión arterial, falla renal y acidosis metabólica persistieron. Con el objetivo de disminuir la demanda metabólica en un contexto de disoxia tisular, es decir un desbalance entre la entrega $\left(\mathrm{DO}_{2}\right)$ y consumo de oxígeno $\left(\mathrm{VO}_{2}\right)$, se indujo hipotermia moderada (rango: $33-34{ }^{\circ} \mathrm{C}$ ) en una búsqueda de descenso del consumo de oxígeno. Para su instauración es necesaria la medición continua de temperatura central (termómetro digital esofágico), así como el uso de bloqueo neuromuscular. En el escenario de cuidados intensivos es importante mantener la hipotermia en un rango moderado (nunca menor a $32^{\circ} \mathrm{C}$ ), ya que al profundizarla aumentan en forma exponencial las complicaciones como trastornos del ritmo cardíaco, de la coagulación y mayor riesgo de infecciones. Dentro del tratamiento no farmacológico del shock cardiogénico grave, un avance significativo se ha producido en las últimas décadas con el desarrollo de dispositivos de soporte mecánico. Sin embargo, en pacientes con ALCAPA, ya que es una lesión con posibilidades de resolver quirúrgicamente habitualmente no son necesarios.

En el ámbito quirúrgico existen varias opciones, como la ligadura de la coronaria en su origen, anastomosis de la coronaria anómala con aorta o subclavia izquierda, operación del túnel intrapulmonar (técnica de takeuchi) donde se une la aorta y la coronaria izquierda ${ }^{13}$. Actualmente es de preferencia la transferencia coronaria directa en la aorta, ya que para mejorar el pronóstico a largo plazo se debe asegurar un sistema coronario binario ${ }^{4,20}$. No es necesario corregir la insuficiencia mitral pues esta es ocasionada por la dilatación del ventrículo izquierdo y del annulus ${ }^{21}$, recuperando función valvular en más del $90 \%$ de los pacientes ${ }^{13,19}$. El resultado a largo plazo es bueno, si se realiza la revascularización en forma temprana. Michielon y cols, reportan una sobrevida de $94 \%$ a los 8 años, dado principalmente por la mortalidad perioperatoria ${ }^{22}$. Los factores de riesgo asociados con mayor mortalidad perioperatoria son: menor edad, evidencias electrocardiográficas de extenso IAM, tipo de circulación coronaria balanceada o izquierda ${ }^{22}$. En un análisis de regresión lineal, este grupo demostró una correlación entre la edad de reparación del defecto y la recuperación de la fracción de eyección, lo que explica otras observaciones y reportes en menores de 6 meses en que la IAACI se presenta en forma grave, con mayor morbimortalidad perioperatoria, pero mejor función miocárdica a largo plazo.

\section{Conclusiones}

La IAACI es una malformación congénita poco frecuente. Es importante tenerla presente como diagnóstico diferencial en cualquier niño 
que presente disnea, precordalgia, miocardiopatía dilatada o arritmia, ya que la precocidad de su diagnóstico influye su tratamiento y pronóstico. La evaluación ecocardiográfica es de gran utilidad, pero ésta debe incluir específicamente la descripción del origen de las arterias coronarias. Al igual que otras malformaciones coronarias hemodinámicamente significativas, su resolución quirúrgica es una emergencia, siendo su pronóstico postoperatorio bueno con las técnicas actuales.

\section{Referencias}

1.- De Wolf D, Vercruysse T, Suys B, Blom N, Matthys D, Ottenkamp J: Major coronary anomalies in childhood. Eur J Pediatr 2002; 161: 637-42.

2.- Basso C, Corrado D, Thiene G: Congenital coronary artery anomalies as an important cause of sudden death in the young. Cardiol Rev 2001; 9: 312-7.

3.- Levin D, Fellows $K$ : Hemodinamically significant primary anomalies of the coronary arteries. Circulation 1978; 58: 25-34.

4.- Chang R, Allada V: Electrocardiographic and echocardiographic features that distinguish anomalous origin of the left coronary artery from pulmonary artery from idiopathic dilated cardiomyopthy. Pediatr Cardiol 2001; 22: 3-10.

5.- Duran AC, Angelini A, Frescura C, Basso C, Thiene G: Anomalous origin of the right coronary artery from the left aortic sinus and sudden infant death. Int $\mathrm{J}$ Cardiol 1994; 45: 147-9.

6.- Frommelt PC, Frommelt MA: Congenital coronary artery anomalies. Pediatr Clin N Am 2004; 51: 1263 88.

7.- Cowles RA, Berdon WE: Bland-white-garland syndrome of anomalous left coronary artery arising from the pulmonary artery (alcapa): a historical review. Pediatr Radiol 2007; 37: 890-5.

8.- Lee A, Foster E, Yeghiazarians Y: Anomalous origin of the left coronary artery from the pulmonary artery: a case series and brief. Congenital Heart Disease 2006; 1: 111-5.

9.- Williams I, Gersony W, Hellenbrand W: Anomalous right coronary artery arising from the pulmonary artery: a report of 7 cases and a review of the literature. American Heart Journal 2006; 152: 1004e917.

10.- Parale GP, Pawar SS: Adult type anomalous left coronary artery from pulmonary artery. J Assoc Physicians India 2006; 54: 397-9.

11.- Grosse-Wortmann L, Wenzl T, Hoevels-Guerich HH: Anomalous origin of the left coronary artery from the pulmonary artery in a premature infant with preserved left ventricular function. Pediatr Cardiol 2006; 27: 269-71.

12.- Werner B, Wroblewska-Kaluzewska M, Pleskot M, Tarnowska A, Potocka K: Anomalies of the coronary arteries in children. Med Sci Monit 2001; 7: 1285-91.

13.- Dahle G, Fiane A, Lindberg H: Alcapa, a posible reason for mitral insufficiency and heart failure in young patients. Scan Cardiovasc Journal 2001; 41: 51-8.

14.- Koike K, Musewe N, Smallhorn J, Freedom R: Distinguishing between anomalous origin of the left coronary artery from the pulmonary trunk and dilated cardiomyopathy: role of echocardiographic measurement of the right coronary artery diameter. Br Heart 1989; 61: 192-7.

15.- Ariza J, Mesa D, Pan M: Anomalous origin of the left coronary artery from the pulmonary artery: diagnosis by transesophageal echocardiography in an infant. Rev Esp Cardiol 2006; 59: 736.

16.- Girish R: Multislice cardiac computed tomographic images of anomalous origin of the left coronary artery from the puulmonary artery. Heart 2006; 92: 2 .

17.- Duerinckx A, Bogaeret J, Jiang H, Lewis B: Anomalous origin of the left coronary artery: diagnosis by coronary $\mathrm{mr}$ angiography. American Journal of Radiology 1995; 164: 1095-7.

18.- Dodge-Khatami A, Mavroudis C, Backer C: Anomalous origin of the left coronary artery from the pulmonary artery: collective review of surgical therapy. Ann Thorac Surg 2002; 74: 946-55.

19.- Babertakis N, Efstathiou A, Esdtathiou N, Papagiannopoulou P, Soulountsi V, Fessatidis I: A longterm survivor of bland-white-garland syndrome with systemic collateral suply: a case report and review of the literature. BMC Surgery 2005; 5: 23-27.

20.- Bunton $R$, Jonas $R A$, Lang $P$, et al: Anomalous origin of the left coronary artery from pulmonary artery: ligation versus establishment of a two coronary artery system. J Thorac Cardiovasc Surg 1987; 93: 103-8.

21.- Schwartz ML, Jonas RA, Colan SD: Anomalous origin of left coronary from pulmonary artery: recovery of left ventricular function after dual coronary repair. J Am Coll Cardiol 1997; 30: 547-53.

22.- Michielon G, Di Carlo D, Branacaccio G, et al: Anomalous coronary artery origin from the pulmonary artery: correlation between surgical timing and left ventricular function recovery. Ann Thorac Surg 2003; 76: $581-8$. 